OPEN ACCESS

Edited by: Gianluca Tozzi, University of Portsmouth, United Kingdom

Reviewed by:

Simone Tassani,

Pompeu Fabra University, Spain Urszula Stachewicz, AGH University of Science and

Technology, Poland

*Correspondence:

Chaozong Liu

chaozong.liu@ucl.ac.uk

Specialty section: This article was submitted to

Mechanics of Materials,

a section of the journal

Frontiers in Materials

Received: 31 July 2017 Accepted: 30 October 2017 Published: 27 November 2017

Citation:

Tamaddon M, Chen SM, Vanaclocha L, Hart A, El-Husseiny M, Henckel J and Liu C (2017) Decrease in Local Volumetric Bone Mineral Density in Osteoarthritic Joints Is Associated with the Increase in Cartilage Damage: A Peripheral

Quantitative CT Study.

Front. Mater. 4:37.

doi: 10.3389/fmats.2017.00037

\section{Decrease in Local Volumetric Bone Mineral Density in Osteoarthritic Joints Is Associated with the Increase in Cartilage Damage: A Peripheral Quantitative CT Study}

\author{
Maryam Tamaddon', Shen Mao Chen', Leyre Vanaclocha', Alister Hart ${ }^{1,2}$, \\ Moataz El-Husseiny ${ }^{2}$, Johann Henckel ${ }^{2}$ and Chaozong Liu ${ }^{1 *}$
}

'Division of Surgery and Interventional Science, Institute of Orthopaedic and Musculoskeletal Science, University College London, Royal National Orthopaedic Hospital, London, United Kingdom, ${ }^{2}$ Royal National Orthopaedic Hospital, London, United Kingdom

Osteoarthritis $(\mathrm{OA})$ is one of the most prevalent joint diseases, which causes pain and disability in the adult population. OA affects the osteochondral unit in the joints, which comprises both cartilage and subchondral bone. There has been some progress in understanding the changes in subchondral bone with progression of OA. However, local changes in subchondral bone such as microstructure or volumetric bone mineral density (vBMD) in connection with the defect in cartilage are relatively unexplored. To develop an effective treatment for progression of $\mathrm{OA}$, it is important to understand how the physical environment provided by the subchondral bone affects the overlying cartilage. In this study, we examined the VBMD distribution in the OA joint tissues obtained from total hip replacement surgeries due to $O A$, using peripheral quantitative CT (pQCT). It was found that there is a significant decrease in $\mathrm{VBMD}$, which co-localizes with the damage in the overlying cartilage. This was not limited to the subchondral bone immediately adjacent to the cartilage defect but continued in the layers below. Bone resorption and cyst formation in the OA tissues were also detected. We observed that the bone surrounding subchondral bone cysts exhibited much higher vBMD than that of the surrounding bones. PQCT was able to detect significant changes in vBMD between OA and non-OA samples, as well as between areas of different cartilage degeneration, which points to its potential as a technique for detection of early $O A$.

Keywords: volumetric bone mineral density, osteoarthritis, subchondral bone, cartilage degeneration, bone cyst, peripheral quantitative $\mathrm{CT}$

\section{INTRODUCTION}

Osteoarthritis $(\mathrm{OA})$ is a degenerative joint disease, characterized by degradation of cartilage and changes in the subchondral bone. Patients with OA often suffer from pain, loss of mobility, and go on to require an end-stage total joint replacement. In 2015, 215,502 joint replacements were performed in England and Wales predominantly for OA (93\%) (National Joint Registry for England, 2015). According to the World Health Organisation, 52\% of people over 70 years old have OA. It affects around 400 million people's lives, and this number will increase with an increasing aging population (Goldring and Goldring, 2010, Litwic et al., 2013). It has been predicted that the 
incidence of OA would be increased to $35 \%$ by 2030 (Thomas et al., 2014). OA affects both articular cartilage and subchondral bone. The degradation and loss of cartilage during OA stems from the decrease in the levels of proteoglycans and changes in biomechanics of the extracellular matrix, altering morphology and metabolic activity of chondrocytes (Martel-Pelletier et al., 2008, Maldonado and Nam, 2013). Radiologically, OA is characterized by the narrowing of joint space, osteophytes formation, subchondral sclerosis, and subchondral bone cysts are usually observed in advanced cases. In OA, thickness of subchondral bone comprising both subchondral bone plate and subarticular spongiosa is increased, mineral content is reduced, and trabecular structure is changed (Madry et al., 2016).

It has been shown that bone mineral density (BMD) changes are associated with the progression of OA (Sepriano et al., 2015). Reliable methods to assess BMD have been attracting an increasing interest. Currently, dual-energy x-ray absorptiometry (DXA) and magnetic resonance imaging (MRI) are used to measure the BMD. However, MRI techniques are only able to provide an indirect measurement of BMD (Bennell et al., 2008). Another technique used for BMD research is quantitative computerized tomography (QCT), which utilizes an X-ray based device to measure BMD in three dimensional space (Adams, 2013). One of the advantages of QCT over the conventional DXA is that the volumetric bone mineral density $\left(\mathrm{vBMD}, \mathrm{mg} / \mathrm{cm}^{3}\right)$ readings obtained from it, is independent of bone size (Bennell et al., 2008). Peripheral quantitative CT (pQCT) is a type of QCT that is increasingly used in measurements of BMD especially in peripheral regions of the body. Although $\mathrm{BMD}$ of the joint is positively associated with prevalence and incidence of $\mathrm{OA}$, its relationship with the advancement of $\mathrm{OA}$ is not well understood (Lee et al., 2013). More so, scarcely data are available regarding the local changes in BMD with progressive degeneration of articular cartilage. The aim of this study was, therefore, to measure and compare the subchondral vBMD distribution in more advanced $\mathrm{OA}$ joints and correlate vBMD distribution with degeneration of overlying articular cartilage. The results would improve our understanding of the role of subchondral bone remodeling and resorption on the cartilage degeneration.

\section{MATERIALS AND METHODS}

\section{Sample Collection}

Femoral heads of nine patients undergoing total hip replacement were collected at Royal National Orthopedic Hospital, approved by UK Health Research Authority (REC reference: 15/LO/2052). Inclusion criteria consisted of femoral heads which had been removed during arthroplasty due to clinically diagnosed OA $(n=7)$ or due to developmental dysplasia of the hip (DDH) (non-OA, $n=1$ ). The non-OA sample is used as a reference for comparison in cartilage grading only. Exclusion criteria included excessive joint damage or deformation that prevented a successful analysis. Age of the patients ranged between 25 and 78 (mean $61.14 \pm 15.7$ ). The characteristics of the patients such as height, body mass index, history of other disease, and medications were not disclosed and were not a concern in this study. Specimens were preserved in $10 \%$ neutral buffered formalin at $4^{\circ} \mathrm{C}$ until required.

\section{Cartilage Grading}

The cartilage on the femoral head was visually graded by two independent observers using modified Outerbridge classification (Wright, 2014) (Table 1). A map was designed containing 36 different regions of interests (ROIs) for analysis of cartilage degeneration of vBMD distribution, as shown in Figure 1A. A non-OA femoral head from a 25 -year-old patient due DDH was used as reference for comparison. The reason for replacement was $\mathrm{DDH}$ but the head did not show any severe cartilage damage.

\section{Measurements of vBMD}

The femoral heads were introduced into a pQCT machine (Stratec XCT3000, Germany) to measure the vBMD $\left(\mathrm{mg} / \mathrm{cm}^{3}\right)$. The samples were scanned through six groups which together gave information on all 36 ROIs (Figure 1A) on three different depth levels: outer (0-5 mm), mid (5-10 mm), and inner $(10-15 \mathrm{~mm})$, as illustrated in Figure 1B. In total, 108 ROIs per sample were examined. The pQCT scans were analyzed using XCT3000 software (version 6.20, Stratec, Germany), which provided direct data on trabecular vBMD (thereafter referred to as $\mathrm{vBMD}$ ). The parameters for the $\mathrm{pQCT}$ scan were set to 10 slices of $0.1 \mathrm{~mm}, 0.2 \mathrm{~mm}$ voxel size, $180 \mathrm{mg} / \mathrm{cm}^{3}$ threshold and trabecular area of $90 \%$. Therefore, in total 1,080 ROIs per sample were obtained, and the vBMD of these 10 slices were averaged and the mean value was used for each of the 108 analyzed ROIs (Figure 1B). On those femoral head scanned where cysts existed, the cysts were excluded in the ROIs as they are filled with fluid, not bone, and would affect the true vBMD calculations (Figure 2).

\section{Statistical Analysis}

For cartilage grading, Kruskal-Wallis tests were used to determine any significant differences between $\mathrm{OA}$ and non-OA samples. For vBMD, effects of the two factors (cartilage grading and depth/level) and their interactions on vBMD were examined. Data were ranked and aligned as Schapiro-Wilk tests rejected normality and a nonparametric multifactorial test was required. Aligned rank transformation (ART) of data was performed using the ARTool software (Kay and Wobbrock, 2017) available at http://depts.washington.edu/madlab/proj/art/ (Wobbrock et al., 2011). Statistical analysis was then performed on ART data using the SPSS 22 (SPSS Inc., Chicago, IL, USA) full-factorial ANOVA. The data were plotted using OriginPro 2017 (OriginLab, Northampton, MA, USA).

TABLE 1 | Outerbridge classification for grading the degeneration of cartilage.

\begin{tabular}{ll} 
Grade & Visual finding \\
\hline 0 & Normal articular cartilage \\
I & Swelling and softening of the articular cartilage \\
II & Fibrillation or superficial fissures of the cartilage \\
III & Deep fibrillation or fissures of the cartilage without exposed bone \\
& Exposure of subchondral bone
\end{tabular}


A

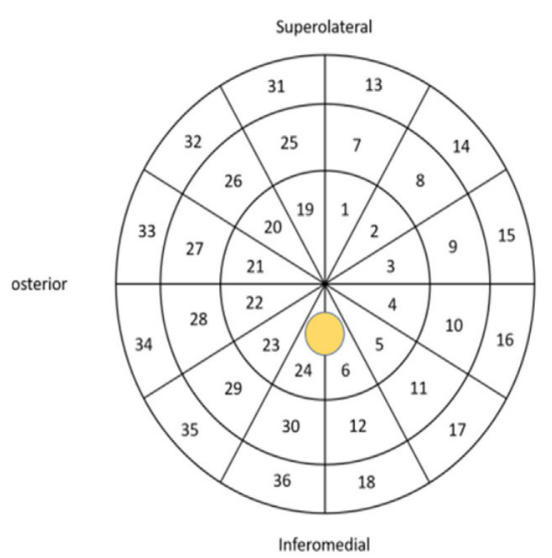

b

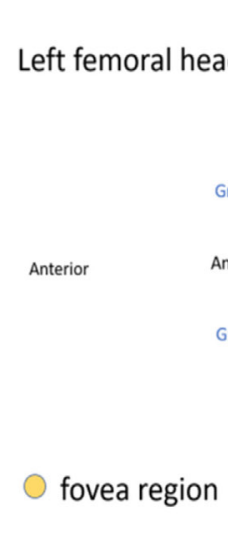

Right femoral head

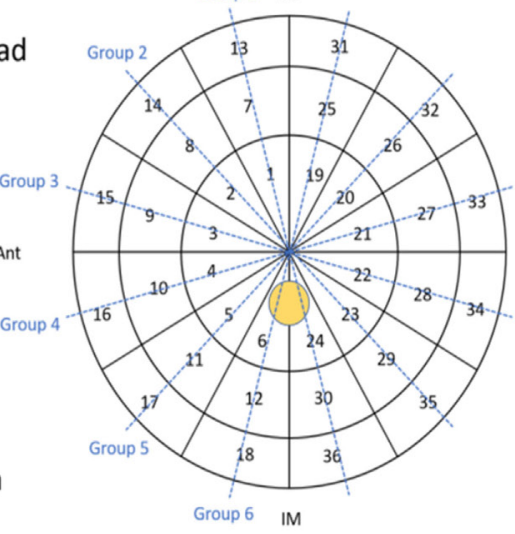

B

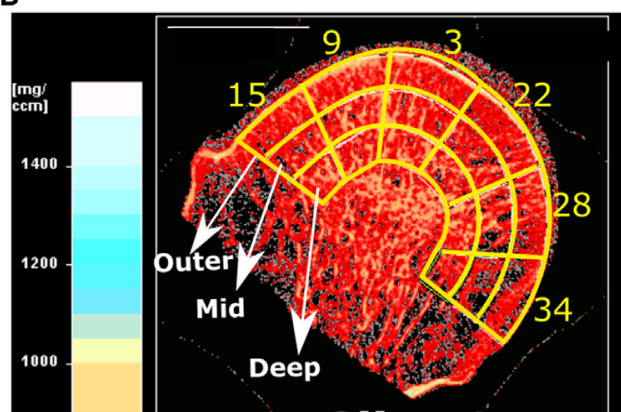

Slice 1

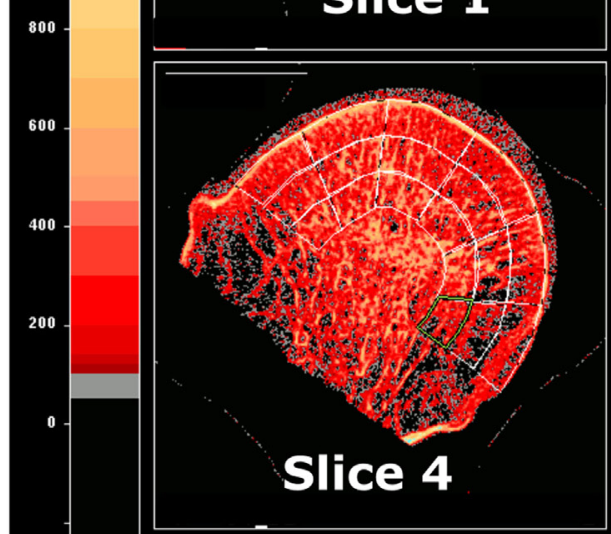

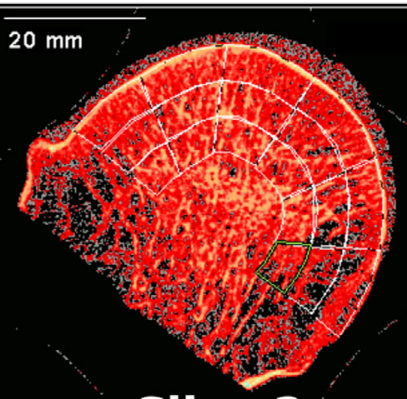

slice 2

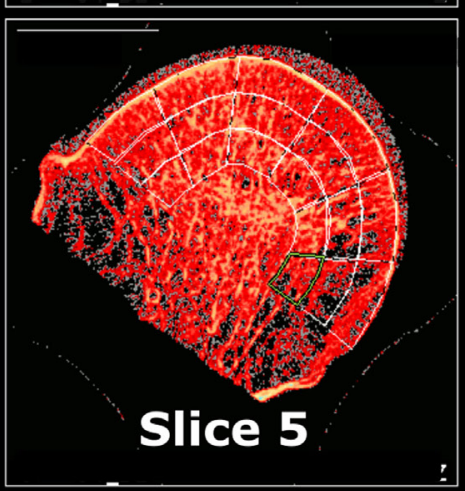

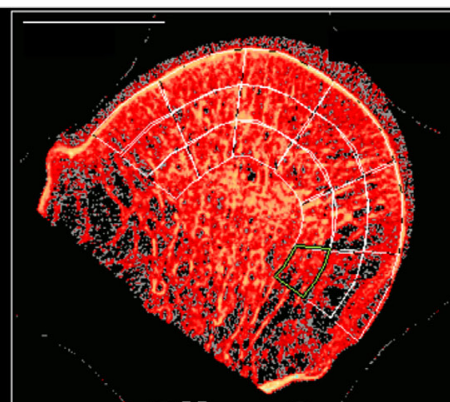

Slice 3

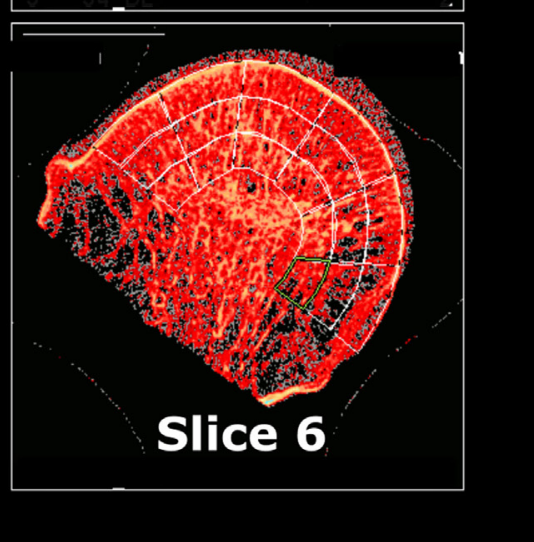

FIGURE 1 | (A) Panel (a) shows the different ROls, birdview from a left femoral head, and panel (b) shows the scan lines on a right femoral head. (B) Shows the three different scan depths across 6 different regions in group 3, which according to panel (a) are regions 15, 9, 3, 22, 28, and 34. 6 out of 10 slices for this group are shown.

\section{RESULTS}

\section{The Changes in Cartilage Grading and Subchondral vBMD with OA}

Cartilage grading with Outerbridge classification were performed in all 36 ROIs of OA samples. It was observed that the remaining cartilage on the femoral heads are in varying degrees of degeneration (I-IV). Whereas in the non-OA control sample no severe (grade III or IV) cartilage degradation was observed. Overall scoring of the cartilage showed that the cartilage on OA femoral heads have significantly higher score than that of control sample $(p<0.001)$. Cartilage degeneration pattern in the OA samples are quite different from that of control DDH sample, as demonstrated in Figure 3. 


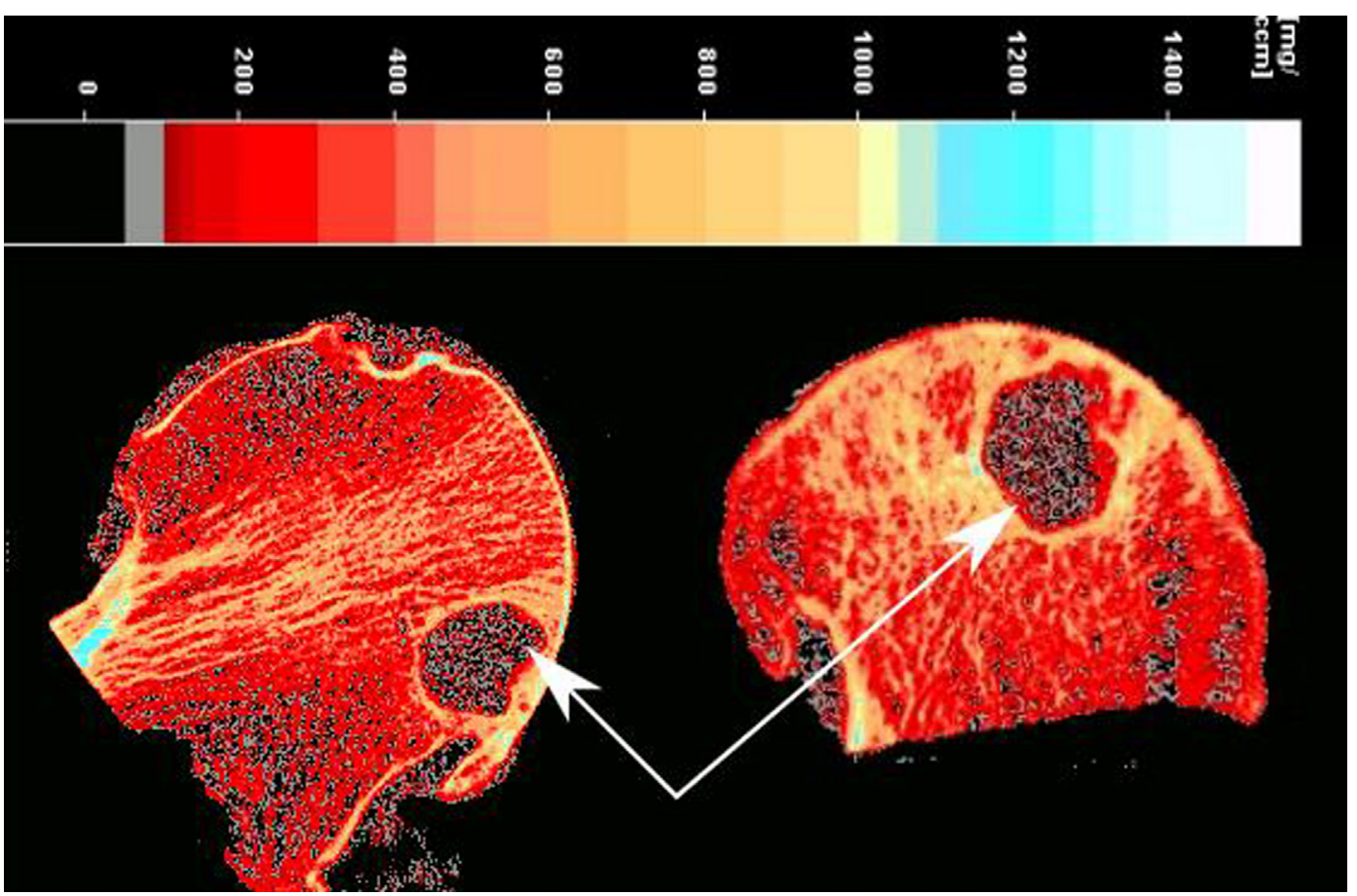

FIGURE 2 | Presence of a large cyst (white arrows) in an osteoarthritis sample.
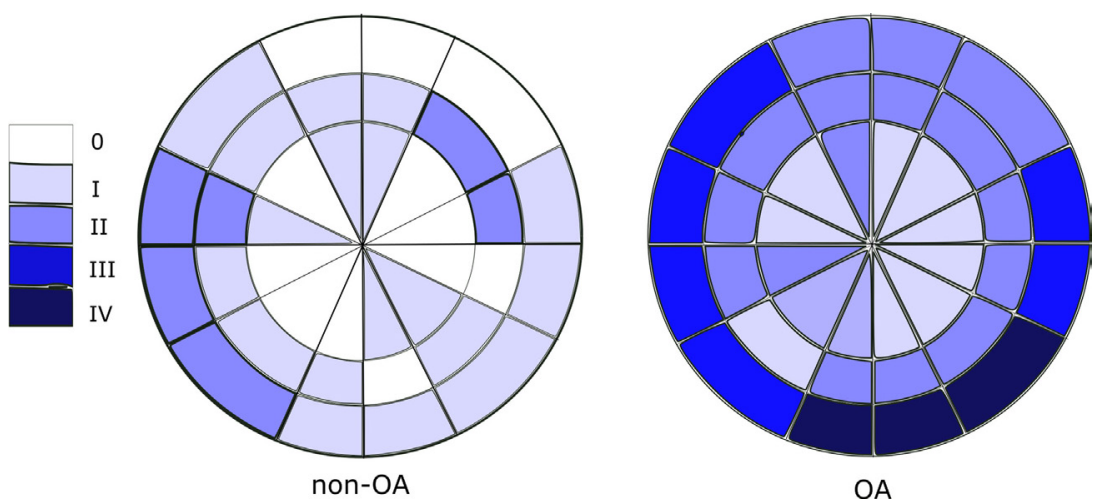

FIGURE 3 | Outerbridge scoring (median score) of OA and non-OA samples shows minimal cartilage degeneration in non-OA samples and severe cartilage degeneration in $25 \%$ of regions of interest in OA samples.

\section{The Effects of Cartilage Degeneration and Depth on Subchondral vBMD}

Both cartilage grading and depth had significant effects on vBMD in the OA group $(p<0.001)$. Within the OA group, there was an inverse relationship between the cartilage grading and vBMD (Figure 4A), meaning that the vBMD decreased with progressively damaged cartilage, which was shown by a weak $(r=-0.36)$, but significant $(p<0.001)$ Pearson correlation between cartilage damage and $v B M D$. Statistical analysis confirmed a significant decrease in vBMD with cartilage damage $(p<0.001)$ with an exception between grade II and III cartilage.
In the outer levels, the subchondral bone adjacent to the grade I cartilage had a vBMD of $423 \mathrm{mg} / \mathrm{cm}^{3}( \pm 161)$; this decreased to $358 \mathrm{mg} / \mathrm{cm}^{3}$ ( \pm 157$)$ for the subchondral bone adjacent to the grade II cartilage, and $333 \mathrm{mg} / \mathrm{cm}^{3}( \pm 143)$ for the bone beneath grade III cartilage. Much lower vBMD values, $246 \mathrm{mg} / \mathrm{cm}^{3}( \pm 96)$, was observed for the subchondral bone adjacent to a more severely degraded grade IV cartilage.

Also, the depth from the cartilage surface significantly affected the vBMD measurements $(p<0.001)$, where mid levels showed significantly lower vBMD compared to outer and inner levels, as shown in Figure 4B. 

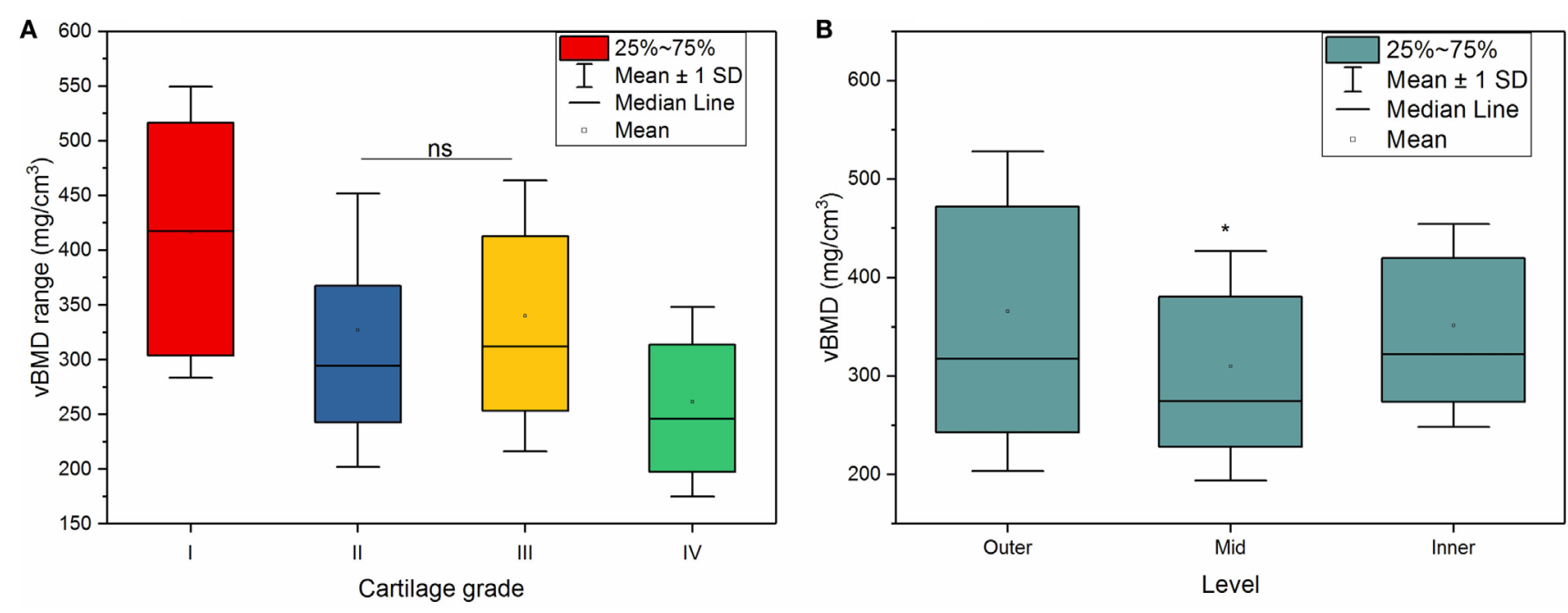

FIGURE 4 | (A) Volumetric bone mineral density (VBMD) of osteoarthritis samples according cartilage grading. A significant decrease in vBMD of subchondral bone with cartilage grading was observed at all grades ( $0<0.001)$, except between grade II and III (ns, no significant difference). (B) vBMD according to depth. Mid levels showed significantly lower vBMD compared to outer and inner levels; ${ }^{*} p<0.001$.

\section{The Combined Effects of Cartilage Degeneration and Depth on Subchondral vBMD}

The decline in vBMD with cartilage grading was observed not only in the outer level, which is adjacent to the overlying cartilage, but also in mid and inner levels, which are distant to the degenerated cartilage as shown in Figure 5.

There was a significant interaction between cartilage degeneration and depth $(p=0.003)$, which means that cartilage degeneration affected vBMD in some levels more than others. In fact, the level with the most change was the outer level $\left(761.01-121.54=639.47 \mathrm{mg} / \mathrm{cm}^{3}\right)$, followed by the middle level $\left(648.73-132.93=515.8 \mathrm{mg} / \mathrm{cm}^{3}\right)$ and finally by the inner depth $\left(658.21-153.72=504.49 \mathrm{mg} / \mathrm{cm}^{3}\right)($ Figure 6A). This shows that the advanced cartilage damage (grade IV) affects outer levels significantly more than mid and inner layers.

Another interesting observation was related to the vBMD surrounding the subchondral bone cysts. These subchondral bone cysts were present in all of OA cases and areas surrounding the cysts showed a significantly higher vBMD $\left(894.8 \pm 64 \mathrm{mg} / \mathrm{cm}^{3}\right)$ compared to the neighboring sites (Figure 2).

\section{DISCUSSION}

We investigated the effects of cartilage degeneration in the OA joints on subchondral vBMD both locally and distantly to the overlying cartilage, as well as the association of vBMD in $\mathrm{OA}$ subchondral bone with the degree of damage in the articular cartilage as assessed through pQCT.

We observed that degradation of cartilage in the OA joints had a significant effect on vBMD distribution in the subchondral bone. In the layer adjacent to the overlying cartilage, the vBMD decreased significantly with cartilage degeneration (Figure 5). This could be explained by the fact that cartilage damage induced

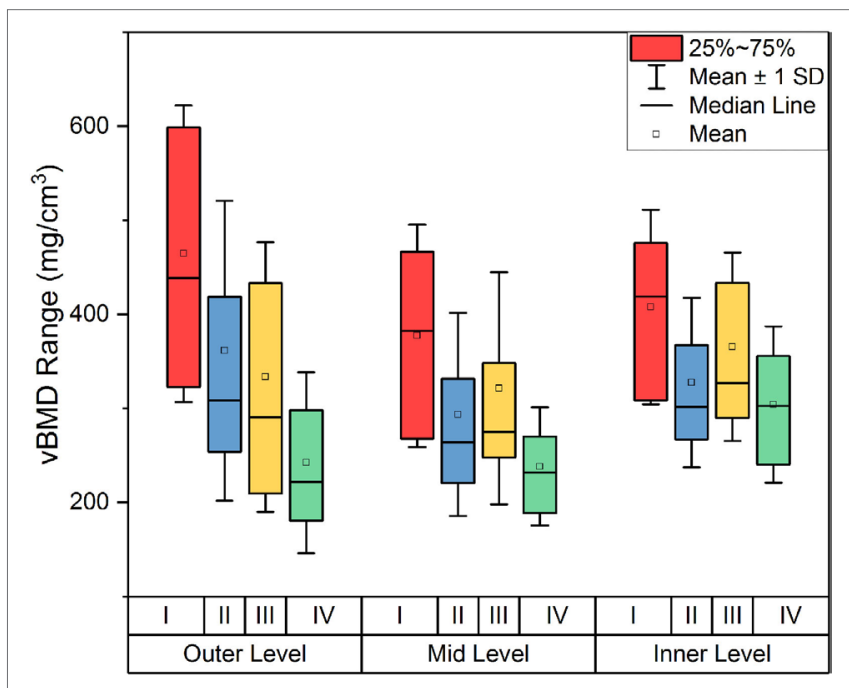

FIGURE 5 | Volumetric bone mineral density (VBMD) of OA samples according to both cartilage grading and depth.

by OA can lead to increased bone remodeling as a result of changes in the loading regime. This may lead to excessive bone turnover, increased bone fraction, and decreased levels of mineralization (Grynpas et al., 1991; Fazzalari and Parkinson, 1997; Stewart et al., 1999; Cox et al., 2012; Yu et al., 2016), all leading to a decreased vBMD. However, the literature is not conclusive on the effects of OA pathology on trabecular density (Karvonen et al., 1998). A study by Dore et al. (2010) suggested that subchondral BMD could not predict cartilage loss, while other studies on OA joints claimed that there is no association between changes in OA grade and changes in BMD (Muehleman et al., 2002; Haugen et al., 2007; Abdin-Mohamed et al., 2009). Nevertheless, we observed a decreased subchondral vBMD with a pattern that 

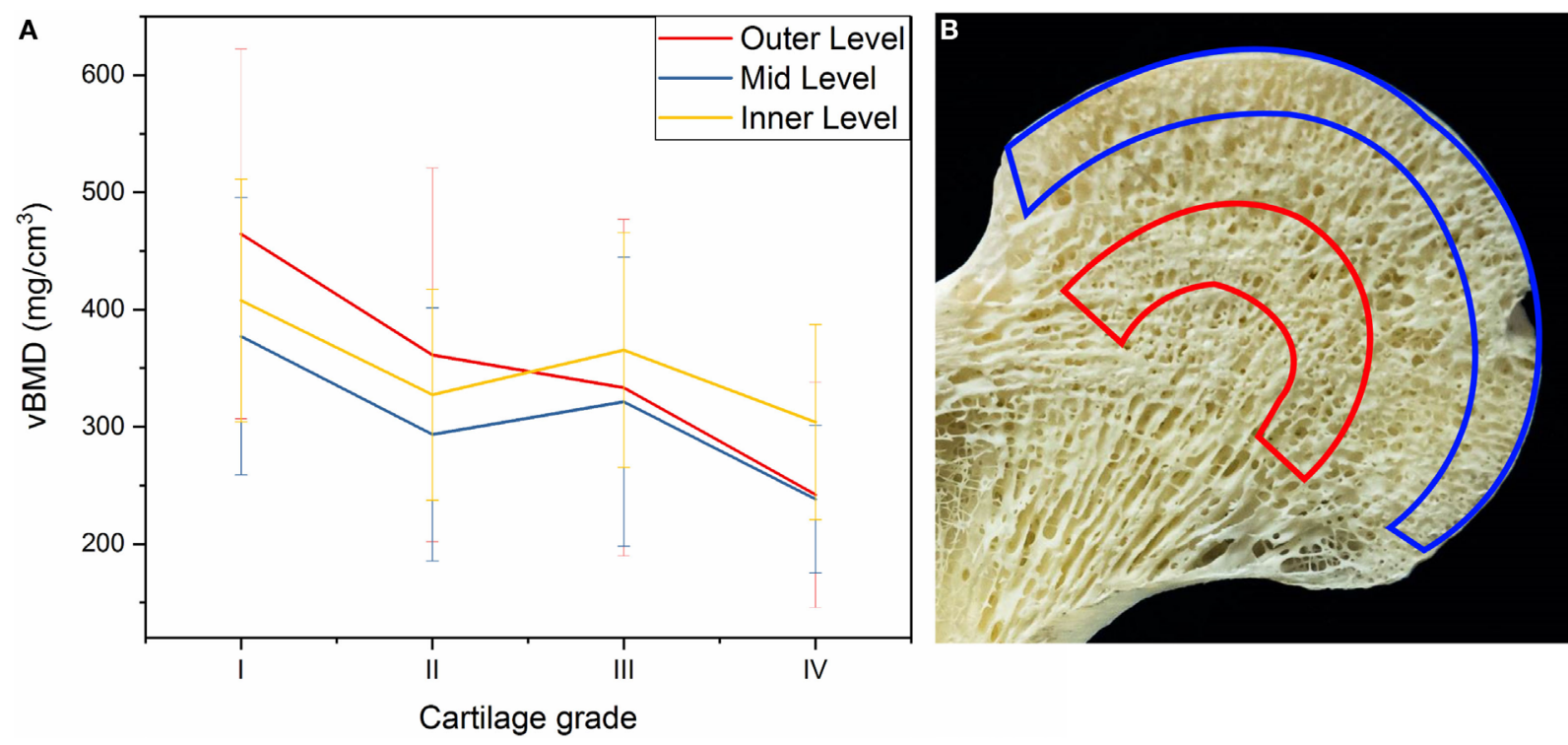

FIGURE 6 | (A) Changes in volumetric bone mineral density (vBMD) of OA femoral heads in outer, mid, and inner levels according to cartilage grading. (B) Outer and inner levels are highlighted (blue and red, respectively) in the femoral head to illustrate the density of each region.

associates with zones of damage in articular cartilage, indicating that the subchondral trabecular vBMD declines with increasing OA pathology, which was shown by the negative correlation of vBMD with Outerbridge Cartilage Scale grade as demonstrated in Figure 4A. This may show the potential of using pQCT for detection of early OA based on changes in vBMD; based on our results, with progression of $\mathrm{OA}$, for example from grade I to grade II, a significant decrease is detectable by pQCT.

A search in the literature would hint to conflicting results regarding $\mathrm{BMD}$ changes with $\mathrm{OA}$. While studying patients affected by $\mathrm{OA}$, some researchers have discovered higher BMD in the joints, while others have not observed the same effects (Sandini et al., 2005). Sandini et al. looked at femoral BMD in subjects with OA and although they found increased bone mineral content and bone size, they did not observe higher BMD in those subjects. This was also accompanied by a faster BMD loss compared with healthy controls. Conversely, Lee et al. (2016) observed that hand and knee OA were negatively associated with the BMDs of the lumbar spine and femoral neck.

These controversies surrounding the relationship between BMD and OA may be explained by the following factors: differences in the measurement methods and differences in the measurement sites. One must note the difference between $\mathrm{vBMD}$ and BMD; the former is a volumetric measurement determined using QCT methods such as pQCT, while the latter is an areal measurement quantified using methods such as DXA. DXA provides a reliable evaluation of areal bone density but is incapable of distinguishing between cortical and trabecular bone (the parameter measured and discussed in this article) or take into account changes in the bone size, which may overestimate the resultant BMD, whereas pQCT can provide accurate measurements of true vBMD and can supply isolated measurement of cortical and trabecular bones
(Abdin-Mohamed et al., 2009). As such, although radiographic OA of the knee is known to be connected to elevated areal BMD at the hip and spine, the vBMD of tibia was not increased in patients with knee OA (Lee et al., 2016).

Another important factor which may cause confusion when comparing $\mathrm{BMD} / \mathrm{vBMD}$ data across different studies is the measurement site (i.e., systemic, distal, or local measurements). For example, Lee et al. showed that BMD loss, measured at a distant site, is associated with progressive loss of cartilage in knees with OA, confirming what was observed by Zhang et al. (2000) that again BMD loss is linked with deterioration of the joint shown by radiographic joint space narrowing. Changes in BMD with OA may differ in different joints. For example, using Raman Spectroscopy it has been shown that the subchondral bone from OA hip joints can have reduced mineral-to-collagen ratio (decreased vBMD), whereas subchondral bone from OA knee joints can have increased mineral-to-collagen ratio (increased vBMD) (Buckley et al., 2014).

The results from our study suggest that vBMD is highest in the outer layer of the trabecular bone, followed by the inner layer, and finally the mid layer as demonstrated in Figure 5B. The outer layer includes the cortical subchondral bone plate which may explain the highest vBMD. The fact that inner layers showed higher vBMD compared to mid layer may be explained according to the trabecular patterns observed by Wolff's Law, where the inner layer in our study coincides with the denser region of where principal compressive and tensile trabecular groups meet as shown in Figure 6B.

The level which experienced the most change with increasing OA grade of the cartilage was the outer level, as shown in Figure $\mathbf{6 A}$, suggesting that the outer layer is the most affected one by the cartilage degeneration in the OA joints and the core in the femoral head was the one least affected. This is possibly 
because OA pathology related changes such as cysts or microcracks occur much more on the surface than in deeper layers. In fact, two studies performed on OA patellae and tibial plateaus in different depths showed similar results and found that the least dense layer was the internal layer (Burnett et al., 2016) and deeper trabecular bone layers have decreased vBMD compared to the surface (Johnston et al., 2011).

We observed subchondral bone cysts in all of OA samples, which are consistent with other reports on OA hip (Chiba et al., 2014). These fluid-filled voids in subchondral bone, which are typically referred to as "subchondral bone cysts," are normally reported in OA patients. There are two main theories about the source of subchondral bone cysts in OA. The "synovial fluid intrusion" theory suggests that due to cracks in the osteochondral region, synovial fluid enters into subchondral bone and leads to development of these cysts; while the "bone contusion" theory proposes that the necrotic lesions in subchondral bone, generated by abnormal mechanical stress result in microcracks, edema, focal bone resorption, and subsequent cyst formation ( $\mathrm{Li}$ et al., 2013). Our study showed that the vBMD of the subchondral bone surrounding the cysts are significantly higher than the neighboring areas, indicating possible bone remodeling as a result of cyst formation. Increased local BMD has also been previously associated with bone marrow lesions in patients with knee OA (Lo et al., 2005), showing a positive relationship between loading and BMD as the response of bone to local stresses.

Further studies in the field could increase the understanding of OA pathogenesis and facilitate the development of new and improved treatments, possibly using pQCT techniques.

\section{CONCLUSION}

The local changes in subchondral vBMD with OA were assessed in OA femoral head using pQCT. We observed a negative association between local vBMD and severity of cartilage damages,

\section{REFERENCES}

Abdin-Mohamed, M., Jameson, K., Dennison, E. M., Cooper, C., and Arden, N. K. (2009). Volumetric bone mineral density of the tibia is not increased in subjects with radiographic knee osteoarthritis. Osteoarthr. Cartil. 17, 174-177. doi:10.1016/j.joca.2008.06.004

Adams, J. E. (2013). Advances in bone imaging for osteoporosis. Nat. Rev. Endocrinol. 9, 28-42. doi:10.1038/nrendo.2012.217

Bennell, K. L., Creaby, M. W., Wrigley, T. V., and Hunter, D. J. (2008). Tibial subchondral trabecular volumetric bone density in medial knee joint osteoarthritis using peripheral quantitative computed tomography technology. Arthritis Rheum. 58, 2776-2785. doi:10.1002/art.23795

Buckley, K., Kerns, J. G., Parker, A. W., Goodship, A. E., and Matousek, P. (2014). Decomposition of in vivo spatially offset Raman spectroscopy data using multivariate analysis techniques. J. Raman Spectrosc. 45, 188-192. doi:10.1002/ jrs. 4434

Burnett, W., Kontulainen, S., McLennan, C., Hazel, D., Talmo, C., Hunter, D., et al. (2016). Patella bone density is lower in knee osteoarthritis patients experiencing moderate-to-severe pain at rest. J. Musculoskelet. Neuronal. Interact. 16, 33-39. doi:10.1016/j.joca.2012.02.326

Chiba, K., Burghardt, A. J., Osaki, M., and Majumdar, S. (2014). Three-dimensional analysis of subchondral cysts in hip osteoarthritis: an ex vivo HR-pQCT study. Bone 66, 140-145. doi:10.1016/j.bone.2014.06.001

Cox, L. G., van Donkelaar, C. C., van Rietbergen, B., Emans, P. J., and Ito, K. (2012). Decreased bone tissue mineralization can partly explain subchondral especially in outer layers of subchondral bone, which we believe is linked to the changes in subchondral bone remodeling. PQCT was able to detect significant changes in vBMD between areas of different cartilage degeneration score, which points to its potential as a technique for detection of early OA.

\section{ETHICS STATEMENT}

All procedures performed in this study involving human participants were approved by the ethical standards of NHS Health Research Authority, London-City \& East Research Ethics Committee (REC reference: 15/LO/2052) and were in accordance with the 1964 Helsinki declaration and its later amendments or comparable ethical standards. Also written informed consent was obtained from all individual participants included in the study.

\section{AUTHOR CONTRIBUTIONS}

MT contributed to the experimental work and prepared the manuscript; SC and LV conducted the experimental work and contributed to drafting the manuscript; $\mathrm{AH}, \mathrm{ME}-\mathrm{H}$, and $\mathrm{JH}$ collected the samples; CL designed the study and contributed to editing of the manuscript. All authors reviewed the paper critically for intellectual content and approved the final version. All authors agreed to be accountable for all aspects of the work in ensuring that questions related to the accuracy or integrity of any part of the work are appropriately investigated and resolved.

\section{FUNDING}

This work was financially supported by The Arthritis Research UK Proof of Concept Award (grant no: 21160) and Rosetrees Trust (project no: A1184).

sclerosis observed in osteoarthritis. Bone 50, 1152-1161. doi:10.1016/j. bone.2012.01.024

Dore, D., Quinn, S., Ding, C., Winzenberg, T., Cicuttini, F., and Jones, G. (2010). Subchondral bone and cartilage damage: a prospective study in older adults. Arthritis Rheum. 62, 1967-1973. doi:10.1002/art.27467

Fazzalari, N. L., and Parkinson, I. H. (1997). Fractal properties of subchondral cancellous bone in severe osteoarthritis of the hip. J. Bone Miner. Res. 12, 632-640. doi:10.1359/jbmr.1997.12.4.632

Goldring, M. B., and Goldring, S. R. (2010). Articular cartilage and subchondral bone in the pathogenesis of osteoarthritis. Ann. N. Y. Acad. Sci. 1192, 230-237. doi:10.1111/j.1749-6632.2009.05240.x

Grynpas, M. D., Alpert, B., Katz, I., Lieberman, I., and Pritzker, K. P. H. (1991). Subchondral bone in osteoarthritis. Calcif. Tissue Int. 49, 20-26. doi:10.1007/ BF02555898

Haugen, I. K., Slatkowsky-Christensen, B., Ørstavik, R., and Kvien, T. K. (2007). Bone mineral density in patients with hand osteoarthritis compared to population controls and patients with rheumatoid arthritis. Ann. Rheum. Dis. 66, 1594-1598. doi:10.1136/ard.2006.068940

Johnston, J. D., McLennan, C. E., Hunter, D. J., and Wilson, D. R. (2011). In vivo precision of a depth-specific topographic mapping technique in the CT analysis of osteoarthritic and normal proximal tibial subchondral bone density. Skeletal Radiol. 40, 1057-1064. doi:10.1007/s00256-010-1001-6

Karvonen, R. L., Miller, P. R., Nelson, D. A., Granda, J. L., and Fernandez-Madrid, F. (1998). Periarticular osteoporosis in osteoarthritis of the knee. J. Rheumatol. $25,2187-2194$ 
Kay, M., and Wobbrock, J. (2017). ARTool: Aligned Rank Transform for Nonparametric Factorial ANOVAs. Version 1.6.2.

Lee, J. Y., Harvey, W. F., Price, L. L., Paulus, J. K., Dawson-Hughes, B., and McAlindon, T. E. (2013). Relationship of bone mineral density to progression of knee osteoarthritis. Arthritis Rheum. 65, 1541-1546. doi:10.1002/art. 37926

Lee, K.-E., Wen, L., Yim, Y.-R., Kim, J.-E., Lee, J.-W., Park, D.-J., et al. (2016). AB0758 the relationships between bone mineral density and radiographic features of hand or knee osteoarthritis in older adults: data from the Dong-Gu study. Ann. Rheum. Dis. 75(Suppl. 2), 1164-1164. doi:10.1136/annrheumdis-2016eular. 1952

Li, G., Yin, J., Gao, J., Cheng, T. S., Pavlos, N. J., Zhang, C., et al. (2013). Subchondral bone in osteoarthritis: insight into risk factors and microstructural changes. Arthritis Res. Ther. 15, 223. doi:10.1186/ar4405

Litwic, A., Edwards, M. H., Dennison, E. M., and Cooper, C. (2013). Epidemiology and burden of osteoarthritis. Br. Med. Bull. 105, 185-199. doi:10.1093/bmb/lds038

Lo, G. H., Hunter, D. J., Zhang, Y., McLennan, C. E., Lavalley, M. P., Kiel, D. P., et al. (2005). Bone marrow lesions in the knee are associated with increased local bone density. Arthritis Rheum. 52, 2814-2821. doi:10.1002/art.21290

Madry, H., Orth, P., and Cucchiarini, M. (2016). Role of the subchondral bone in articular cartilage degeneration and repair. J. Am. Acad. Orthop. Surg. 24, e45-e46. doi:10.5435/jaaos-d-16-00096

Maldonado, M., and Nam, J. (2013). The role of changes in extracellular matrix of cartilage in the presence of inflammation on the pathology of osteoarthritis. Biomed. Res. Int. 2013, 284873. doi:10.1155/2013/284873

Martel-Pelletier, J., Boileau, C., Pelletier, J.-P., and Roughley, P. J. (2008). Cartilage in normal and osteoarthritis conditions. Best Prac. Res. Clin. Rheumatol. 22, 351-384. doi:10.1016/j.berh.2008.02.001

Muehleman, C., Berzins, A., Koepp, H., Eger, W., Cole, A. A., Kuettner, K. E., et al. (2002). Bone density of the human talus does not increase with the cartilage degeneration score. Anat. Rec. 266, 81-86. doi:10.1002/ar.10040

National Joint Registry for England, Wales and Northern Ireland. (2015). NJR StatsOnline. Available at: http://www.njrcentre.org.uk/njrcentre/LinkClick.aspx? fileticket $=\mathrm{QkZM} 7 \mathrm{sN}-\mathrm{HpA} \% 3 \mathrm{~d} \&$ portalid $=0$

Sandini, L., Arokoski, J. P. A., Jurvelin, J. S., and Kröger, H. (2005). Increased bone mineral content but not bone mineral density in the hip in surgically treated knee and hip osteoarthritis. J. Rheumatol. 32, 1951-1957.
Sepriano, A., Roman-Blas, J. A., Little, R. D., Pimentel-Santos, F., Arribas, J. M. Largo, R., et al. (2015). DXA in the assessment of subchondral bone mineral density in knee osteoarthritis - a semi-standardized protocol after systematic review. Semin. Arthritis Rheum. 45, 275-283. doi:10.1016/j.semarthrit.2015.06.012

Stewart, A., Black, A., Robins, S. P., and Reid, D. M. (1999). Bone density and bone turnover in patients with osteoarthritis and osteoporosis. J. Rheumatol. 26, 622-626.

Thomas, E., Peat, G., and Croft, P. (2014). Defining and mapping the person with osteoarthritis for population studies and public health. Rheumatology (Oxford) 53, 338-345. doi:10.1093/rheumatology/ket346

Wobbrock, J. O., Findlater, L., Gergle, D., and Higgins, J. J. (2011). “The aligned rank transform for nonparametric factorial analyses using only ANOVA procedures," in Proceedings of the ACM Conference on Human Factors in Computing Systems (CHI '11). Vancouver, British Columbia. New York: ACM Press, 143-146.

Wright, R. W. (2014). Osteoarthritis classification scales: interobserver reliability and arthroscopic correlation. J. Bone Joint Surg. Am. 96, 1145-1151. doi:10.2106/ jbjs.m.00929

Yu, D., Xu, J., Liu, F., Wang, X., Mao, Y., and Zhu, Z. (2016). Subchondral bone changes and the impacts on joint pain and articular cartilage degeneration in osteoarthritis. Clin. Exp. Rheumatol. 34, 929-934.

Zhang, Y., Hannan, M. T., Chaisson, C. E., McAlindon, T. E., Evans, S. R., Aliabadi, P., et al. (2000). Bone mineral density and risk of incident and progressive radiographic knee osteoarthritis in women: the Framingham Study. J. Rheumatol. 27, 1032-1037.

Conflict of Interest Statement: The authors declare that the research was conducted in the absence of any commercial or financial relationships that could be construed as a potential conflict of interest.

Copyright $\odot 2017$ Tamaddon, Chen, Vanaclocha, Hart, El-Husseiny, Henckel and Liu. This is an open-access article distributed under the terms of the Creative Commons Attribution License (CC BY). The use, distribution or reproduction in other forums is permitted, provided the original author(s) or licensor are credited and that the original publication in this journal is cited, in accordance with accepted academic practice. No use, distribution or reproduction is permitted which does not comply with these terms. 